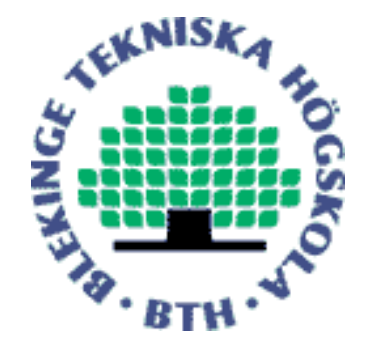

Copyright (C) 2014 IEEE.

Citation for the published paper:

Adaptive Modulation and Coding with Queue Awareness in Cognitive Incremental Decode-and-Forward Relay Networks

Thi My Chinh Chu, Hoc Phan, Hans-Jürgen Zepernick

IEEE International Conference on Communications

2014 Sydney, Australia

This material is posted here with permission of the IEEE. Such permission of the IEEE does not in any way imply IEEE endorsement of any of BTH's products or services Internal or personal use of this material is permitted. However, permission to reprint/republish this material for advertising or promotional purposes or for creating new collective works for resale or redistribution must be obtained from the IEEE by sending a blank email message to pubs-permissions@iee.org.

By choosing to view this document, you agree to all provisions of the copyright laws protecting it. 


\title{
Adaptive Modulation and Coding with Queue Awareness in Cognitive Incremental Decode-and-Forward Relay Networks
}

\author{
Thi My Chinh Chu, Hoc Phan, and Hans-Jürgen Zepernick \\ Blekinge Institute of Technology, SE-371 79 Karlskrona, Sweden \\ E-mail: \{cch,hph,hjz\}@bth.se
}

\begin{abstract}
This paper studies the performance of adaptive modulation and coding in a cognitive incremental decode-andforward relaying network where a secondary source can directly communicate with a secondary destination or via an intermediate relay. To maximize transmission efficiency, a policy which flexibly switches between the relaying and direct transmission is proposed. In particular, the transmission, which gives higher average transmission efficiency, will be selected for the communication. Specifically, the direct transmission will be chosen if its instantaneous signal-to-noise ratio (SNR) is higher than one half of that of the relaying transmission. In this case, the appropriate modulation and coding scheme (MCS) of the direct transmission is selected only based on its instantaneous SNR. In the relaying transmission, since the MCS of the transmissions from the source to the relay and from the relay to the destination are implemented independently to each other, buffering of packets at the relay is necessary. To avoid buffer overflow at the relay, the MCS for the relaying transmission is selected by considering both the queue state and the respective instantaneous SNR. Finally, a finite-state Markov chain is modeled to analyze key performance indicators such as outage probability and average transmission efficiency of the cognitive relay network.
\end{abstract}

\section{INTRODUCTION}

The recent thriving in wireless communications has dramatically increased the scarcity of radio spectrum. However, measurement campaigns have shown that spectrum bands using a fixed spectrum allocation policy are often under-utilized. This necessitates new technologies to improve the efficiency of spectrum utilization. A novel idea of opportunistically utilizing particular bands of the spectrum, called cognitive radios, was introduced by Mitola [1], [2]. This spectrum-allocation policy allows cognitive users, also called secondary users (SUs), to coexist with licensed users or primary users (PUs) in the same spectrum band without degrading the performance of the PUs. To satisfy this requirement, the SU, while accessing the spectrum band, must always consider the impact of its transmission on the quality of the PU reception. In the spectrum-sharing context, [3] has introduced the interference temperature concept and the tolerable interference level at the primary receiver. Therein, the SU is considered not to affect the PU's performance if the interference power at the primary receiver is kept below a predefined acceptable threshold. Because of this rigid spectrum access regulation, the SU must strictly constrain its transmit power and hence communication range in the secondary network is very limited.
In order to overcome this limitation of spectrum-sharing, relaying transmission has been considered as a potential solution to increase the radio coverage [4]. Based on how the signal is processed at the relay, there are two well-known relaying schemes, namely, decode-and-forward (DF) relaying and amplify-and-forward (AF) relaying. In an AF relay system, the signal is simply amplified at the relay and then forwarded to the destination. Thus, not only the desired signal but also both noise and interference are amplified at the relay. On the contrary, in the DF relay system, the received signal from the source is decoded, regenerated at the relay and then transmitted to the destination. Since only the desired signal is forwarded to the destination, DF relaying is suitable for transmission in interference environments. This, in turn, seems applicable to spectrum-sharing cognitive radio networks where the secondary transmitter is required to emit its signals with rather low power to meet the interference power constraint at the primary receiver.

The policies to select the relaying transmission are distinguished as fixed, selection, and incremental relaying [5]. The simplest scheme is the fixed relaying where the relays are always utilized regardless of their performance. Thus, the fixed relaying suffers from a reduction in spectrum efficiency. Based upon fixed relaying, the selection relaying scheme allows a source to select cooperation or non-cooperation with a suitable relay. By continuously comparing the instantaneous signal-tonoise ratios (SNRs) between the links, the source can select the relay which offers the highest SNR to forward the signal to the destination. With incremental relaying, the relaying mode is active only if the instantaneous SNR raises beyond a predefined threshold. Because of limiting the amount of feedback in terms of channel state information (CSI), the spectral efficiency of the incremental relaying outperforms that of fixed and selection relaying [5]. An alternative method to enhance spectrum efficiency is to deploy appropriate link adaptation strategies where certain parameters such as transmit power, modulation scheme, code rate or any combination of these parameters are adjusted to the variations of the fading channel [6]. Furthermore, this strategy conserves transmit power to reduce the interference from its transmission to the PU as well as to alleviate the effect of the fading channels. However, since the fading conditions of the transmissions from the source to the relay and from the relay to the destination are 
independent to each other, the modulation and coding schemes for these transmissions may be different. Therefore, a buffer is necessary at the relay to reduce the dropping rate of packets at the relay.

In this paper, we deploy adaptive modulation and coding (AMC) for cognitive incremental DF relay networks where the communication can be performed by the relaying or direct transmission to maximize transmission efficiency. The switching policy for selecting between the relaying and the direct communication is based on [7], i.e., the relaying mode is chosen only if its instantaneous SNR is two times higher than that of the direct mode. Furthermore, we address the overflow issue of the buffer at the relay by considering the queue state when selecting the modulation and coding scheme (MCS) for the relaying transmission. A finite-state Markov chain model is utilized to analyze the distribution of the number of packets in the buffer of the relay which enables us to evaluate outage probability and transmission efficiency of the network.

Notation: In this paper, bold lower case and upper case letters are used to represent vector and matrix, respectively. Next, the probability density function (PDF) and the cumulative distribution function (CDF) of a random variable $X$ are denoted as $f_{X}(\cdot)$ and $F_{X}(\cdot)$, respectively. Furthermore, the ceiling operator and expectation operator are, respectively, expressed as $\lceil\cdot\rceil$ and $E\{\cdot\}$. The binomial operator is represented as $(\cdot)$. Finally, the gamma function [8, eq. (8.310.1)] and the incomplete gamma function [8, eq. (8.350.2)] are represented as $\Gamma(n)$ and $\Gamma(n, x)$, respectively.

\section{System And Channel Model}

The considered cognitive relay network consists of a secondary source $\mathrm{S}$, a secondary relay $\mathrm{R}$, and a secondary destination $\mathrm{D}$. This network operates under the interference power constraint $Q$ of a primary receiver, $\mathrm{P}$, as depicted in Fig. 1. Here, $\mathrm{h}_{\mathrm{yz}}, \mathrm{y} \in\{\mathrm{s}, \mathrm{r}, \mathrm{d}\}, \mathrm{z} \in\{\mathrm{r}, \mathrm{d}, \mathrm{p}\}$, and $\mathrm{y} \neq \mathrm{z}$, is the channel coefficient of the link from $Y \in\{S, R, D\}$ to $\mathrm{Z} \in\{\mathrm{R}, \mathrm{D}, \mathrm{P}\}, \mathrm{Y} \neq \mathrm{Z}$. In order to maximize transmission efficiency in the secondary network, we employ incremental DF relaying where $\mathrm{S}$ can either directly communicate with $\mathrm{D}$ or communicate via the relay R. Selection of the operation mode, direct transmission or relaying, is based on the respective instantaneous SNR. Thus, R and D need to estimate their

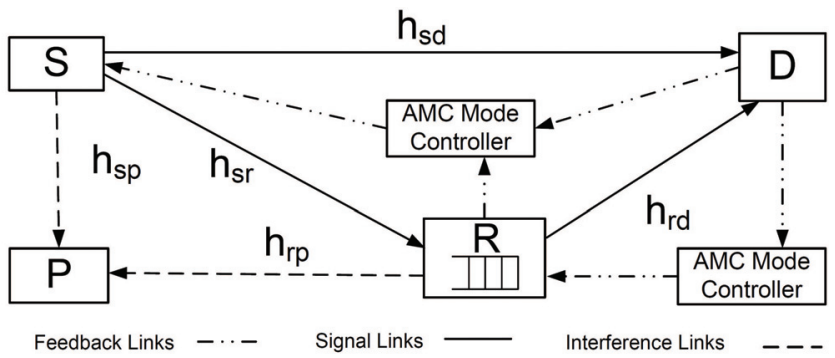

Fig. 1. System model of a cognitive incremental decode-and-forward relay network with adaptive modulation and coding. received SNRs and feed them back to $\mathrm{S}$ and $\mathrm{R}$ to choose the appropriate operation mode. Assume that this network operates under block Nakagami- $m$ fading such that the channels can be considered as constant for the transmit period of each packet. Let $\mathrm{X}_{\mathrm{yz}}$ be the channel power gain corresponding to the channel coefficient $\mathrm{h}_{\mathrm{yz}}$. Also, let us denote $P_{\max }$ as the transmit power limit of both S and R. Under the interference power constraint $Q$ of $\mathrm{P}$ and transmit power limit, $P_{\max }$, of $\mathrm{S}$ and $\mathrm{R}$, the transmit power $P_{s}$ of $\mathrm{S}$ and $P_{r}$ of $\mathrm{R}$ must be controlled as

$$
P_{s}=\min \left(P_{\max }, \frac{Q}{X_{s p}}\right), P_{r}=\min \left(P_{\max }, \frac{Q}{X_{r p}}\right)
$$

Based on [9, eq. (5)], we can derive the CDF, $F_{\gamma_{y z}}(\gamma)$, and PDF, $f_{\gamma_{y z}}(\gamma)$, of the instantaneous SNR $\gamma_{y z}$ of the link with channel coefficient $h_{y z}$ as (2) and (3), respectively. Here, $\beta=P_{\max } / N_{0}, \mu=Q / N_{0}$, and $N_{0}$ is the noise power at the secondary relay and destination. Further, $\alpha_{y z}$ is defined as $\alpha_{y z}=m_{y z} / \Omega_{y z}$ where $m_{y z}$ and $\Omega_{y z}$ are, respectively, the fading severity parameter and channel mean power of the link with channel coefficient $h_{y z}$. Then, the instantaneous SNR of the DF relaying link can be approximated as in [10, eq. (25)]

$$
\gamma_{s r d}=\min \left(\gamma_{s r}, \gamma_{r d}\right)
$$

Then, the CDF, $F_{\gamma_{s r d}}(\gamma)$, of the instantaneous SNR of the relaying link is given by

$$
F_{\gamma_{s r d}}(\gamma)=1-\left[1-F_{\gamma_{s r}}(\gamma)\right]\left[1-F_{\gamma_{r d}}(\gamma)\right]
$$

where $F_{\gamma_{s r}}(\gamma)$ and $F_{\gamma_{r d}}(\gamma)$ are defined in (2) with parameter sets $\left(m_{y z}, \alpha_{y z}, m_{y p}, \alpha_{y p}\right)=\left(m_{s r}, \alpha_{s r}, m_{s p}, \alpha_{s p}\right)$ and $\left(m_{y z}, \alpha_{y z}, m_{y p}, \alpha_{y p}\right)=\left(m_{r d}, \alpha_{r d}, m_{r p}, \alpha_{r p}\right)$, respectively.

In order to improve transmission efficiency, we apply AMC with five different MCSs as shown in Table I [11]. Specifically, the SNR range is divided into 5 regions with the switching thresholds between MCSs given by

$$
\begin{aligned}
& \gamma_{n}=\frac{1}{g_{n}} \ln \left(\frac{a_{n}}{P E R_{T G}}\right), n=1,2, \ldots, N \\
& \gamma_{N+1}=\infty
\end{aligned}
$$

where $a_{n}, g_{n}$ are predefined constants given in Table I, $N$ is the number of modes, in our case, $N=5$, and $P E R_{T G}$ is the target packet error rate. The parameters $a_{n}, g_{n}$ and $\gamma_{T_{n}}$ for the $n$-th mode are obtained by applying the fitting algorithm given in [12]. Assuming that the $n$-th MCS is selected, then there

TABLE I

Modulation and Coding Schemes [11]

\begin{tabular}{|c||c|c|c|c|c|}
\hline & MCS 1 & MCS 2 & MCS 3 & MCS 4 & MCS 5 \\
\hline \hline Modulation & BPSK & QPSK & QPSK & $16-$-AM & $16-$ QAM \\
\hline Code & $1 / 2$ & $1 / 2$ & $3 / 4$ & $9 / 16$ & $3 / 4$ \\
\hline Rate (bps) & 0.50 & 1.00 & 1.50 & 2.25 & 3.00 \\
\hline$a_{n}$ & 274.72 & 90.25 & 67.61 & 50.12 & 53.39 \\
\hline$g_{n}$ & 7.99 & 3.49 & 1.68 & 0.66 & 0.37 \\
\hline$\gamma_{T_{n}}$ & -1.533 & 1.049 & 3.972 & 7.702 & 10.249 \\
\hline
\end{tabular}




$$
\begin{aligned}
& F_{\gamma_{y z}}(\gamma)=1-\sum_{l=0}^{m_{y z}-1} \frac{1}{l !} \frac{\alpha_{y z}^{l} \gamma^{l}}{\beta^{l}} \exp \left(-\frac{\alpha_{y z} \gamma}{\beta}\right)+\sum_{i=0}^{m_{y p}-1} \frac{\alpha_{y p}^{i}}{i !} \alpha_{y z}^{m_{y z}} \mu^{i} \exp \left(-\frac{\alpha_{y p} \mu}{\beta}\right) \frac{\Gamma\left(m_{y z}+i\right)}{\Gamma\left(m_{y z}\right)} \frac{\gamma^{m_{y z}}}{\left(\alpha_{y z} \gamma+\alpha_{y p} \mu\right)^{m_{y z}+i}} \\
& \times \sum_{j=0}^{m_{y z}+i-1} \frac{1}{j !} \frac{\left(\alpha_{y z} \gamma+\alpha_{y p} \mu\right)^{j}}{\beta^{j}} \exp \left(-\frac{\alpha_{y z} \gamma}{\beta}\right)
\end{aligned}
$$

$$
\begin{aligned}
& f_{\gamma_{y z}}(\gamma)=\sum_{l=0}^{m_{y z}-1} \frac{\alpha_{y z}^{l+1}}{\beta^{l+1}} \frac{\gamma^{l}}{l !} \exp \left(-\frac{\alpha_{y z} \gamma}{\beta}\right)-\sum_{l=0}^{m_{y z}-1} \frac{l}{l !} \frac{\alpha_{y z}^{l}}{\beta^{l}} \gamma^{l-1} \exp \left(-\frac{\alpha_{y z} \gamma}{\beta}\right)-\sum_{i=0}^{m_{y p}-1} \frac{\mu^{i}}{i !} \frac{\Gamma\left(m_{y z}+i\right)}{\Gamma\left(m_{y z}\right)} \exp \left(-\frac{\alpha_{y p} \mu}{\beta}\right) \\
& \times \sum_{j=0}^{m_{y z}+i-1} \frac{\alpha_{y p}^{i} \alpha_{y z}^{j-i}}{j ! \beta^{j}} \frac{\gamma_{y z}^{m_{y}}}{\left(\gamma+\alpha_{y p} \mu / \alpha_{y z}\right)^{m_{y z}+i-j}} \exp \left(-\frac{\alpha_{y z} \gamma}{\beta}\right)\left(-\frac{m_{y z}}{\gamma}+\frac{\alpha_{y z}}{\beta}+\frac{m_{y z}+i-j}{\gamma+\alpha_{y p} \mu / \alpha_{y z}}\right)
\end{aligned}
$$

are $n$ packets transmitted during one time slot, $T_{s}$. However, if the instantaneous SNRs of both the direct and relaying links fall below $\gamma_{1}$, the system falls into outage and no transmission takes place. As the transmission extends over two time slots, the overall transmission efficiency of the relaying mode is reduced by half [13]. Thus, the relaying mode is chosen only if the instantaneous SNR over the relaying link, $\gamma_{s r d}$, is two times higher than that of the direct link, $\gamma_{s d}$, i.e., $\gamma_{s d}<\frac{\gamma_{s r d}}{2}$.

In the direct communication, i.e. $\gamma_{s d} \geq \frac{\gamma_{s r d}}{2}, \mathrm{~S}$ chooses an appropriate number of packets with fixed length, encodes and modulates them with a suitable MCS from Table I and then transmits the signal to $\mathrm{D}$ while $\mathrm{R}$ remains silent. The MCS selection for the direct transmission is only based on the fading condition of the link from $\mathrm{S}$ to $\mathrm{D}$. In particular, the $n$-th MCS will be assigned to the direct transmission if the instantaneous SNR of the direct transmission $\gamma_{s d}$ falls into the region $\left(\gamma_{n}, \gamma_{n+1}\right)$ and the instantaneous SNR of the relaying transmission $\gamma_{s r d}$ is lower than $\gamma_{2 n+1}$. For this reason, when $\gamma_{\left\lceil\frac{N}{2}\right\rceil} \leq \gamma_{s d}$, there exists only the direct transmission.

In the relaying communication, i.e. $\gamma_{s d}<\frac{\gamma_{s r d}}{2}$, the MCS for the relaying transmission is selected based on both the fading conditions and the state of the buffer at $\mathrm{R}$. This means that $\mathrm{S}$ and $\mathrm{R}$ not only adapt their transmission to the fading condition but also to the current state of the buffer in order to avoid buffer overflow.

We first look at the MCS selection at the source $S$ for the transmission from $\mathrm{S}$ to R. During the first time slot, $\mathrm{S}$ selects an appropriate number of packets, encodes and modulates them by using a suitable MCS and then transmits the result to R. Specifically, the MCS at S is selected based on the fading condition of the channel from $\mathrm{S}$ to $\mathrm{R}$ and the available vacant positions at the buffer of the relay as follows. Assume that there are $N_{e}$ vacant positions available at the buffer of $\mathrm{R}$ for a certain time instant. Furthermore, at that time, the instantaneous SNR $\gamma_{s r}$ of the transmission from $\mathrm{S}$ to $\mathrm{R}$ is assumed to be able to support up to the $t$-th mode, i.e., the instantaneous SNR $\gamma_{s r}$ falls into the below region

$$
\begin{cases}\gamma_{t} \leq \gamma_{\mathrm{sr}}<\gamma_{t+1} & \text { for } t \in\{1,2,3,4\} \\ \gamma_{t} \leq \gamma_{\mathrm{sr}} & \text { for } t=5\end{cases}
$$

In this case, the $n$-th MCS, $n=\min \left(N_{e}, t\right)$, is selected for the transmission from $\mathrm{S}$ to $\mathrm{R}$. Then, $\mathrm{R}$ attempts to demodulate and decode the received signal, places the successfully decoded packets into the buffer which provides $L$ positions for storing packets. It is assumed that the unsuccessfully decoded packets are simply dropped and the higher layers are responsible for detecting and retransmitting these packets.

We are now looking at the MCS selection at the relay for the transmission from $\mathrm{R}$ to $\mathrm{D}$. During the second time slot, $\mathrm{R}$ encodes and modulates an appropriate number of packets using the selected MCS and forwards the resulting signal to D. The MCS selection at R is based on the instantaneous SNR $\gamma_{r d}$ of the transmission from $\mathrm{R}$ to $\mathrm{D}$ and the available packets in the buffer at the relay. Assume that there are $N_{a}$ available packets at the buffer of R and the instantaneous SNR $\gamma_{r d}$ of the transmission from $\mathrm{R}$ to $\mathrm{D}$ falls into the region of the $k$-th mode, i.e.,

$$
\begin{cases}\gamma_{k} \leq \gamma_{\mathrm{rd}}<\gamma_{k+1} & \text { for } k \in\{1,2,3,4\} \\ \gamma_{k} \leq \gamma_{\mathrm{rd}} & \text { for } k=5\end{cases}
$$

Then, the $n$-th MCS, $n=\min \left(N_{a}, k\right)$, is assigned to the transmission from $\mathrm{R}$ to $\mathrm{D}$.

\section{QUEUEING ANALYSis}

Denote $p_{i, j}$ as the transition probability that the number of packets at the buffer of the relay changes from $i$ in the current time interval to $j$ in the next time interval. In order to calculate $p_{i, j}$, we define two following probabilities.

First, we denote $a_{k, i}$ as the probability that, given $i, 0 \leq$ $i \leq L$ packets in its buffer, $\mathrm{R}$ forwards $k, 0 \leq k \leq \min \{i, N\}$, packets to D. It is noted that if $k<i, a_{k, i}$ is the probability that $\gamma_{k} \leq \gamma_{r d} \leq \gamma_{k+1}$. On the contrary, if $k=i, a_{k, i}$ is the probability that the instantaneous SNR $\gamma_{r d}$ of the transmission 
from $\mathrm{R}$ to $\mathrm{D}$ is sufficient to operate in the $k$-th MCS, i.e.,

$$
a_{k, i}= \begin{cases}F_{\gamma_{r d}}\left(\gamma_{k+1}\right)-F_{\gamma_{r d}}\left(\gamma_{k}\right), & k<i \\ 1-F_{\gamma_{r d}}\left(\gamma_{k}\right), & k=i \\ 0, & k>i\end{cases}
$$

Second, we define $b_{h, q}$ as the probability that $h$ new decoded packets are put into the buffer conditioned on having $q$ empty positions in the buffer. This is also the probability that $\mathrm{R}$ successfully decodes the $h$ packets sent from S given $q$ vacant positions in the buffer, i.e.,

$$
b_{h, q}=\sum_{v=h}^{\min (N, q)} p_{h, v, q}
$$

where $p_{h, v, q}$ is the joint probability that $\mathrm{S}$ transmits $v$ packets and R successfully decodes $h$ packets given $q$ empty positions in the buffer. Clearly, if $v<q$, then $p_{h, v, q}$ is the probability that the instantaneous SNR of the link from $\mathrm{S}$ to $\mathrm{R}$ falls in the region of the $v$-th MCS, $\gamma_{v} \leq \gamma_{s r} \leq \gamma_{v+1}$, and exactly $h$ packets are successfully decoded from $v$ transmitted packets. However, if $v=q$, then $p_{h, v, q}$ is the probability that the instantaneous SNR $\gamma_{s r}$ is sufficient for $\mathbf{S}$ to operate in the $v$-th MCS, $\gamma_{s r} \geq \gamma_{v}$, and $h$ packets are successfully decoded from $v$ transmitted packets. As a result, we have

$$
p_{h, v, q}= \begin{cases}\int_{\gamma_{v}}^{\gamma_{v}} s_{h, v}(\gamma) f_{\gamma_{s r}}(\gamma) d \gamma, & v<\min (N, q) \\ \int_{\gamma_{v}}^{\infty} s_{h, v}(\gamma) f_{\gamma_{s r}}(\gamma) d \gamma, & v=\min (N, q) \\ 0, & v>\min (N, q)\end{cases}
$$

Here, $s_{h, v}(\gamma)$ is the probability that R successfully decodes $h$ packets from $v$ transmitted packets from $\mathrm{S}$. Assuming that the probability of packets being successfully decoded is statistical independent from each other, we have

$$
s_{h, v}(\gamma)=\left(\begin{array}{l}
v \\
h
\end{array}\right)\left[1-P_{e, v}(\gamma)\right]^{h} P_{e, v}^{v-h}(\gamma)
$$

where, $P_{e, v}(\gamma)$ is the packet error rate (PER) with the received SNR $\gamma$ when the transmission operates with the $v$-th MCS. As in [12], $P_{e, v}(\gamma)$ is approximated as

$$
P_{e, v}(\gamma)= \begin{cases}1, & 0<\gamma<\gamma_{T_{v}} \\ a_{v} \exp \left(-g_{v} \gamma\right), & \gamma \geq \gamma_{T_{v}}\end{cases}
$$

Substituting (14) into (13), we obtain $s_{h, v}(\gamma)$. Then, substituting this outcome and $f_{\gamma_{s r}}(\gamma)$ defined in (3) into (12) together with the help of [8, eq. (3.381.3)], $p_{h, v, q}$ is given by

$$
p_{h, v, q}= \begin{cases}\theta_{1}(h, v), & v<\min (N, q) \\ \theta_{2}(h, v), & v=\min (N, q) \\ 0, & v>\min (N, q)\end{cases}
$$

where $\theta_{1}(h, v)$ and $\theta_{2}(h, v)$ are given by (16) and (17), respectively. Here, $\Upsilon(a, b, c, d), \xi(a, b)$, and $\phi(a, b, c, d, e)$ are, respectively, defined as

$$
\begin{array}{r}
\Upsilon(a, b, c, d)=\frac{\Gamma\left(a, \frac{\alpha_{1}+\beta(b+c-d) g_{c}}{\beta} \gamma_{c}\right) \beta^{a}}{\left\{\alpha_{1}+\beta(b+c-d) g_{c}\right\}^{a}} \\
-\frac{\Gamma\left(a, \frac{\alpha_{1}+\beta(b+c-d) g_{c}}{\beta} \gamma_{c+1}\right) \beta^{a}}{\left\{\alpha_{1}+\beta(b+c-d) g_{c}\right\}^{a}} \\
\xi(a, b, c, d)=\frac{\Gamma\left(a, \frac{\alpha_{1}+\beta(b+c-d) g_{c}}{\beta} \gamma_{c}\right) \beta^{a}}{\left\{\alpha_{1}+\beta(b+c-d) g_{c}\right\}^{a}} \\
\phi(a, b, c, d, e)=\int_{a}^{\infty} \frac{\gamma^{b}}{(\gamma+c)^{d}} \exp (-e \gamma) d \gamma
\end{array}
$$

Substituting (15) into (11), $b_{h, q}$ is determined.

With the derived expressions of $b_{h, q}$ and $a_{k, i}$, we are now ready to calculate the transition probability $p_{i, j}$ that the number of packets at the buffer of the relay changes from $i$ in the current time interval to $j$ in the next time interval. It can be seen that $p_{i, j}$ is the joint probability that $\mathrm{R}$ forwards $k, 0 \leq k \leq \min \{i, N\}$, packets to $\mathrm{D}$ given $i$ packets in its buffer, $0 \leq i \leq L$, and that $\mathrm{R}$ successfully decodes extra $(j-i+k)$ packets sent from $\mathrm{S}$ given $(L-i+k)$ vacant positions in the buffer, i.e.,

$$
p_{i, j}=\sum_{k=0}^{\min \{i, N\}} a_{k, i} b_{j-i+k, L-i+k}
$$

By substituting the expressions of $a_{k, i}$ in (10) and $b_{h, q}$ in (11) into (21), we finally obtain the transition probability that the buffer of the relay changes from $i$ packets in the current time interval to $j$ packets in the next interval, $p_{i, j}$.

Let $\mathbf{p}=\left(p_{0}, p_{1}, \ldots, p_{L}\right)$ be a vector which represents the distribution of the number of packets in the buffer of $\mathrm{R}$. Here, each component, $p_{k}$ of $\mathbf{p}$, stands for the steady-state probability that there are $k \in\{0, \ldots, L\}$ packets in the buffer at R, i.e., $p_{k} \geq 0$ and $\sum_{i=0}^{L} p_{i}=1$. In order to obtain $\mathbf{p}$, we construct an $(L+1) \times(L+1)$ transition probability matrix A where the element at the $i$-th row and the $j$-th column of $\mathbf{A}$ is $p_{i, j}$. Then, $\mathbf{p}$ is obtained as the solution of the following equation:

$$
\mathbf{p A}=\mathbf{p}
$$

Note that $\mathbf{p}$ is a normalized left eigenvector of $\mathbf{A}$ associated with the eigenvalue one. Utilizing the method of eigenvalue decomposition, performed by the support of mathematical software packages, we obtain the left eigenvector of A corresponding to eigenvalue one. Then, we normalize this vector such that all entries sum up to one to obtain $\mathbf{p}$.

\section{Performance Analysis}

\section{A. Outage Probability}

For the considered system utilizing AMC, if the instantaneous SNRs of both the direct and relaying links fall below the switching threshold $\gamma_{1}$, no transmission occurs. Thus, the 


$$
\begin{aligned}
& \theta_{1}(h, v)=\left(\begin{array}{l}
v \\
h
\end{array}\right) \sum_{\eta=0}^{h}\left(\begin{array}{l}
h \\
\eta
\end{array}\right)(-1)^{\eta} a_{v}^{\eta+v-h}\left\{\sum_{l=0}^{m_{s r}-1} \frac{\alpha_{s r}^{l+1} \Upsilon(l+1, \eta, v, h)}{\beta^{l+1}} \frac{\sum_{l=0}^{m_{s r}-1} \frac{l \Upsilon(l, \eta, v, h)}{l !} \frac{\alpha_{s r}^{l}}{\beta^{l}}+\sum_{i=0}^{m_{s p}-1} \frac{\mu^{i}}{i !} \exp \left(-\frac{\alpha_{s p} \mu}{\beta}\right)}{\times \frac{\Gamma\left(m_{s r}+i\right)}{\Gamma\left(m_{s r}\right)} \sum_{j=0}^{m_{s r}+i-1} \frac{1}{j !} \frac{\alpha_{s p}^{i} \alpha_{s r}^{j-i}}{\beta j}\left\{m _ { s r } \left[\phi\left(\gamma_{v}, m_{s r}-1, \frac{\alpha_{s p} \mu}{\alpha_{s r}}, m_{s r}+i-j, \frac{\alpha_{s r}+\beta(\eta+v-h) g_{v}}{\beta}\right)-\phi\left(\gamma_{v+1}, m_{s r}-1,\right.\right.\right.}\right. \\
& \left.\left.\frac{\alpha_{s p} \mu}{\alpha_{s r}}, m_{s r}+i-j, \frac{\alpha_{s r}+\beta(\eta+v-h) g_{v}}{\beta}\right)\right]-\frac{\alpha_{s r}}{\beta}\left[\phi\left(\gamma_{v}, m_{s r}, \frac{\alpha_{s p} \mu}{\alpha_{s r}}, m_{s r}+i-j, \frac{\alpha_{s r}+\beta(\eta+v-h) g_{v}}{\beta}\right)-\phi\left(\gamma_{v+1}, m_{s r},\right.\right. \\
& \left.\frac{\alpha_{s p} \mu}{\alpha_{s r}}, m_{s r}+i-j, \frac{\alpha_{s r}+\beta(\eta+v-h) g_{v}}{\beta}\right]-\left(m_{s r}+i-j\right)\left[\phi\left(\gamma_{v}, m_{s r}, \frac{\alpha_{s p} \mu}{\alpha_{s r}}, m_{s r}+i+1-j, \frac{\alpha_{s r}+\beta(\eta+v-h) g_{v}}{\beta}\right)\right. \\
& \left.\left.\left.-\phi\left(\gamma_{v+1}, m_{s r}, \frac{\alpha_{s p} \mu}{\alpha_{s r}}, m_{s r}+i-j+1, \frac{\alpha_{s r}+\beta(\eta+v-h) g_{v}}{\beta}\right)\right]\right\}\right\}
\end{aligned}
$$

$$
\begin{aligned}
& \theta_{2}(h, v)=\left(\begin{array}{l}
v \\
h
\end{array}\right) \sum_{\eta=0}^{h}\left(\begin{array}{l}
h \\
\eta
\end{array}\right)(-1)^{\eta} a_{v}^{\eta+v-h}\left\{\sum_{l=0}^{m_{s r}-1} \frac{\xi(l+1, \eta, v, h)}{l !} \frac{\alpha_{s r}^{l+1}}{\beta^{l+1}}-\sum_{l=0}^{m_{s r}-1} \frac{l \xi(l, \eta, v, h)}{l !} \frac{\alpha_{s r}^{l}}{\beta^{l}}+\sum_{i=0}^{m_{s p}-1} \frac{\mu^{i}}{i !} \exp \left(-\frac{\alpha_{s p} \mu}{\beta}\right)\right. \\
& \times \frac{\Gamma\left(m_{s r}+i\right)}{\Gamma\left(m_{s r}\right)} \sum_{j=0}^{m_{s r}+i-1} \frac{\alpha_{s p}^{i} \alpha_{s r}{ }^{j-i}}{j ! \beta j}\left[m_{s r} \phi\left(\gamma_{v}, m_{s r}-1, \frac{\alpha_{s p} \mu}{\alpha_{s r}}, m_{s r}+i-j, \frac{\alpha_{s r}+\beta(\eta+v-h) g_{v}}{\beta}\right)-\frac{\alpha_{s r}}{\beta} \phi\left(\gamma_{v}, m_{s r},\right.\right. \\
& \left.\left.\left.\frac{\alpha_{s p} \mu}{\alpha_{s r}}, m_{s r}+i-j, \frac{\alpha_{s r}+\beta(\eta+v-h) g_{v}}{\beta}\right)-\left(m_{s r}+i-j\right) \phi\left(\gamma_{v}, m_{s r}, \frac{\alpha_{s p} \mu}{\alpha_{s r}}, m_{s r}+i+1-j, \frac{\alpha_{s r}+\beta(\eta+v-h) g_{v}}{\beta}\right)\right]\right\}
\end{aligned}
$$

outage probability $P_{\text {out }}$ of the system is calculated as

$$
P_{\text {out }}=F_{\gamma_{s d}}\left(\gamma_{1}\right) F_{\gamma_{s r d}}\left(\gamma_{1}\right)
$$

where $F_{\gamma_{s d}}(\gamma)$ and $F_{\gamma_{s r d}}(\gamma)$ are given in (2) and (5), respectively.

\section{B. Average Transmission Efficiency}

Recall that incremental relaying is deployed in the considered system. Thus, the average transmission efficiency of the system, i.e., the average number of packets transmitted during one transmission interval, includes average transmission efficiency of the direct and relaying modes. Since packets can be discarded at the relay if they cannot be successfully decoded, the average transmission efficiency of the relaying mode corresponds to the average transmission efficiency of the transmission from $\mathrm{R}$ to $\mathrm{D}$ [11]. Furthermore, in the relay mode, the transmission period is divided in two equal time slots, one to transmit a signal from $\mathrm{S}$ to $\mathrm{R}$ and the other to forward a signal from $\mathrm{R}$ to $\mathrm{D}$. Thus, the transmission efficiency of the relaying mode is reduced to one half as compared to the average transmission efficiency of the transmission from $\mathrm{R}$ to $\mathrm{D}$. As a result, the overall average transmission efficiency of the system is obtained as

$$
\eta=\sum_{n=1}^{N} n P_{s d}(n)+\frac{1}{2} \sum_{t=1}^{N} t P_{r d}(t)
$$

where $P_{s d}(n)$ and $P_{r d}(t)$ are the probabilities that the direct transmission operates using the $n$-th MCS and the transmission of the relay-to-destination link operates using the $t$-th MCS.
Again, the direct transmission will operate using the $n$-th MCS if its instantaneous SNR $\gamma_{s d}$ falls in the operation region of the $n$-th MCS and is higher than one half of the instantaneous SNR $\gamma_{s r d}$ of the relaying transmission. Thus, $P_{s d}(n)$ is given by $P_{s d}(n)=\operatorname{Pr}\left\{\gamma_{n} \leq \gamma_{s d} \leq \gamma_{n+1}, \gamma_{s r d} \leq \gamma_{2 n+1}\right\}$, i.e.,

$$
P_{s d}(n)=\left\{\begin{array}{lc}
F_{\gamma_{s r d}}\left(\gamma_{2 n+1}\right)\left(F_{\gamma_{s d}}\left(\gamma_{n+1}\right)-F_{\gamma_{s d}}\left(\gamma_{n}\right)\right), \\
F_{\gamma_{s d}}\left(\gamma_{n+1}\right)-F_{\gamma_{s d}}\left(\gamma_{n}\right), & 1 \leq n \leq\left\lceil\frac{N}{2}-1\right\rceil \leq n \leq(N-1) \\
1-F_{\gamma_{s d}}\left(\gamma_{N}\right), & n=N
\end{array}\right.
$$

On the other hand, the relay transmission will be chosen only if the instantaneous SNR of the direct link, $\gamma_{s d}$, is lower than one half of the instantaneous SNR of the relaying link, $\gamma_{s r d}$. Specifically, there are two situations that $\mathrm{R}$ will operate using the $t$-th MCS, $0 \leq t \leq 5$. The fist situation occurs when having more than $t$ packets in the buffer and the instantaneous SNR of the link from $\mathrm{R}$ to $\mathrm{D}, \gamma_{r d}$, falls in the operation region of the $t$-th MCS, i.e., $\gamma_{t} \leq \gamma_{r d} \leq \gamma_{t+1}$. The second situation occurs when having exactly $t$ packets in the buffer of $\mathrm{R}$ and the instantaneous SNR of the link from R to D, $\gamma_{r d}$, is sufficient to support the $t$-th MCS, i.e., $\gamma_{t} \leq \gamma_{r d}$. Therefore, $P_{r d}(t)$ is given by (26).

Finally, by substituting (26) and (25) into (24), the overall transmission efficiency of the considered system is obtained.

\section{NumericAl Results AND Discussions}

In this section, we illustrate the effect of the interference power constraint, $Q$, transmit power limit, $P_{\max }$, and packet 


$$
P_{r d}(m)=\left\{\begin{array}{r}
F_{\gamma_{s d}}\left(\gamma_{\lceil m / 2\rceil}\right)\left[F_{\gamma_{s r d}}\left(\gamma_{m+1}\right)-F_{\gamma_{s r d}}\left(\gamma_{m}\right)\right]\left[\left(F_{\gamma_{r d}}\left(\gamma_{m+1}\right)-F_{\gamma_{r d}}\left(\gamma_{m}\right)\right) \sum_{i=m+1}^{L} \pi_{i}+\left(1-F_{\gamma_{r d}}\left(\gamma_{m}\right)\right) \pi_{m}\right], \\
F_{\gamma_{s d}}\left(\gamma_{\lceil m / 2\rceil}\right)\left[1-F_{\gamma_{s r d}}\left(\gamma_{m}\right)\right]\left[\left(F_{\gamma_{r d}}\left(\gamma_{m+1}\right)-F_{\gamma_{r d}}\left(\gamma_{m}\right)\right) \sum_{i=m+1}^{L} \pi_{i}+\left(1-F_{\gamma_{r d}}\left(\gamma_{m}\right)\right) \pi_{m}\right], \quad m=N
\end{array}\right.
$$

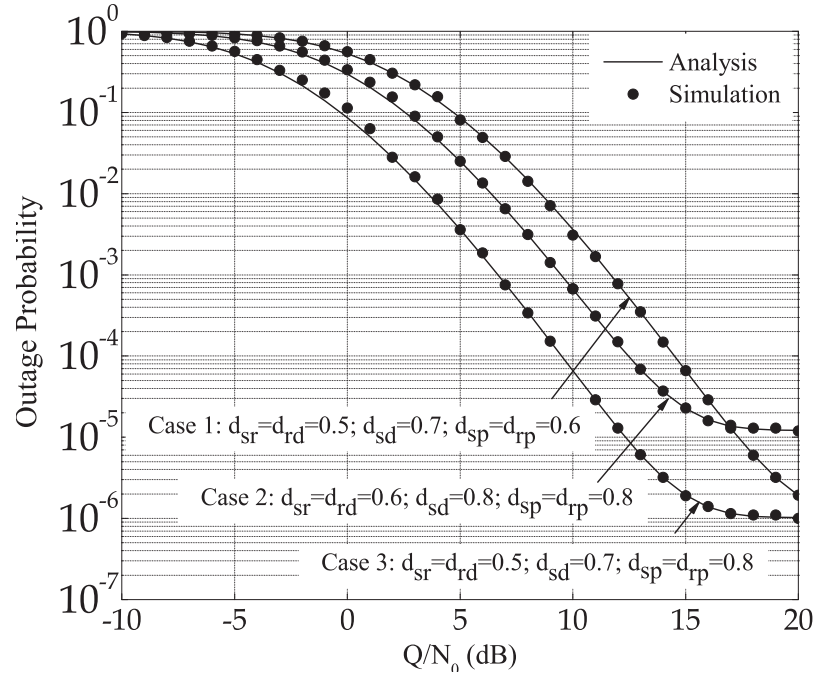

Fig. 2. Outage probability of the secondary network versus interference power-to-noise ratio $Q / N_{0}$ for various transmission distances.

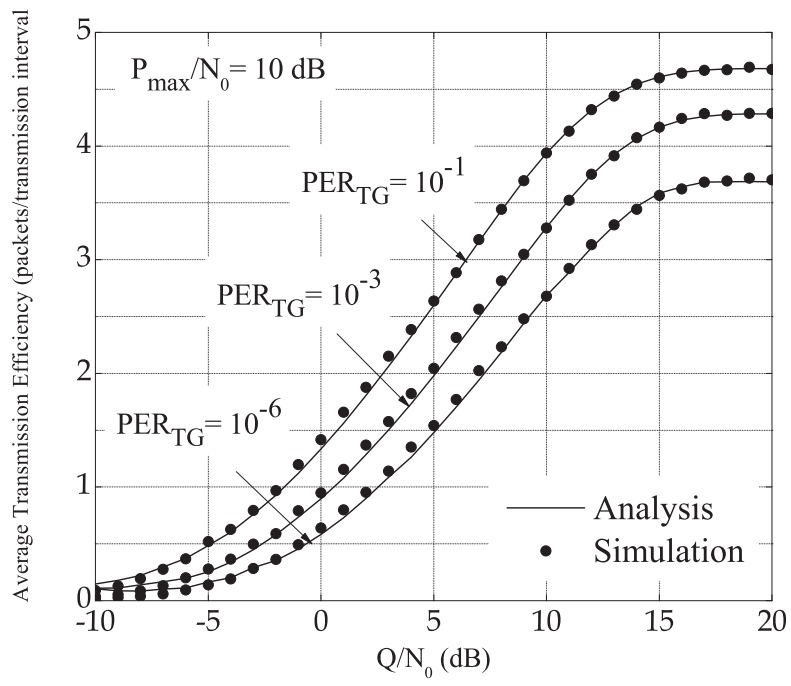

Fig. 3. Transmission efficiency of the secondary network versus interference power-to-noise ratio $Q / N_{0}$ for various packet error rate targets.

error rate target, $P E R_{T G}$, on the outage probability and transmission efficiency of the considered system. The fading severity parameters of all links are selected as $m_{s r}=m_{r d}=$ $m_{s d}=m_{s p}=m_{r p}=2$. Further, we choose the buffer length at $\mathrm{R}$ as $L=5$. Denote $d_{y z}, y \in\{s, r, d\}, z \in\{r, d, p\}$ and $y \neq z$ in Fig. 1 as the transmission distance of the link with channel coefficient $h_{y z}$. We assume that the signal power decays relative to these distances with path-loss exponent 4 .

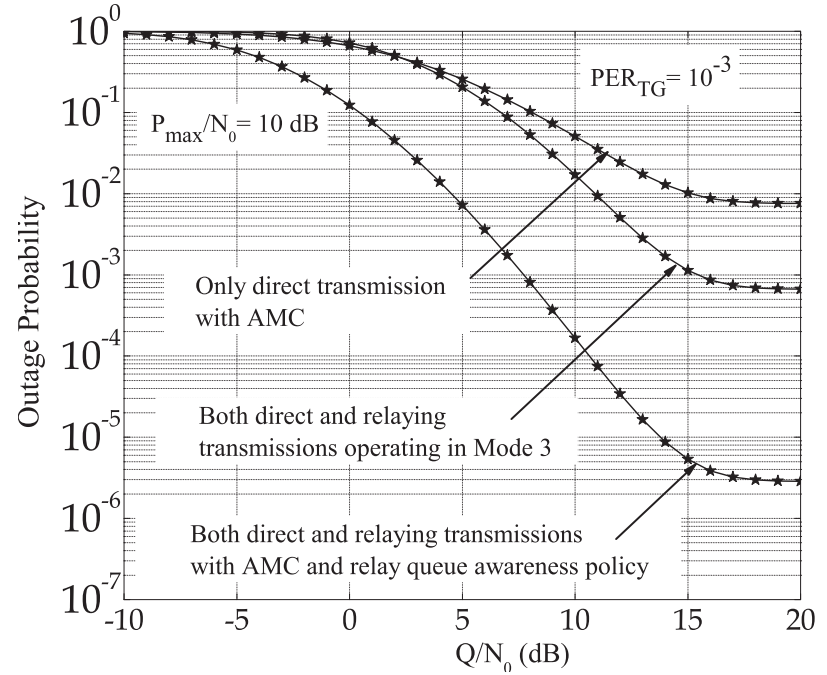

Fig. 4. A comparison of outage probability of the considered system utilizing $\mathrm{AMC}$ and relay queue awareness with that of a system without $\mathrm{AMC}$ and that of the direct transmission utilizing AMC.

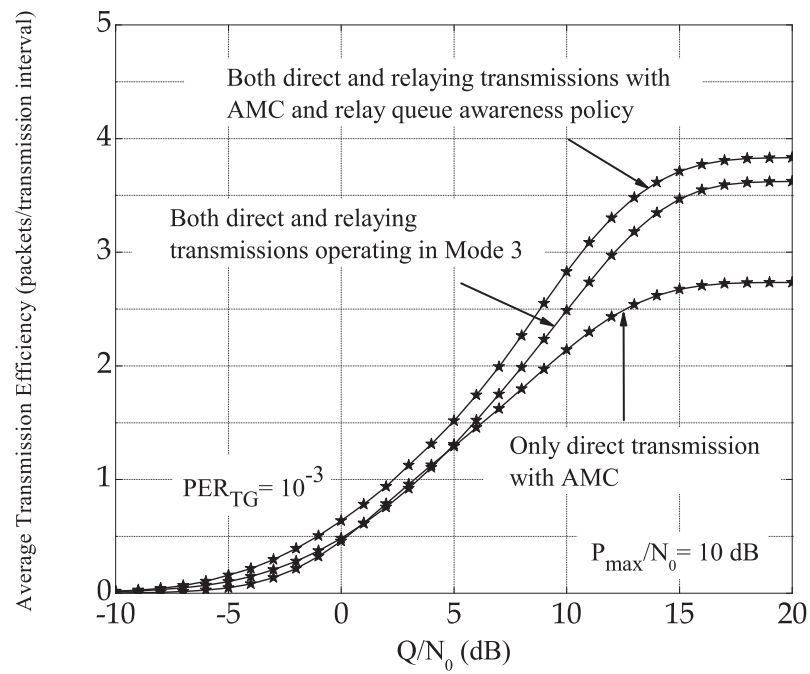

Fig. 5. A comparison of transmission efficiency of the considered system utilizing $\mathrm{AMC}$ and relay queue awareness with that of a system without $\mathrm{AMC}$ and that of the direct transmission utilizing AMC.

Firstly, in Fig. 2, we investigate the impact of the transmission distances on the outage probability of the system. The transmit power limit-to-noise ratio of $\mathrm{S}$ and $\mathrm{R}$ is fixed as $P_{\max } / N_{0}=10 \mathrm{~dB}$ and the packet error rate target, $P E R_{T G}$, is $10^{-3}$. As can be seen from Case 1 and Case 3 of Fig. 2, given the same transmission distances, $\left(d_{s r}, d_{r d}, d_{s d}\right)$, 
of the secondary network, the further the distances of the interference links to the primary user, $\left(d_{s p}, d_{r p}\right)$, the lower the outage probability of the secondary network. This is due to the fact that when the distances of the interference links increase, the constraint on the transmit power of S and R can be more relaxed. Furthermore, by fixing the distances of the interference links in Case 2 and Case 3, we can observe that the outage probability decreases with respect to the decrease in the distances between the terminals in the secondary network.

Secondly, in Fig. 3, we show the impact of $P E R_{T G}$ on the transmission efficiency of the system. For these examples, we have chosen the transmit power limit-to-noise ratio of $\mathrm{S}$ and $\mathrm{R}$ as $P_{\max } / N_{0}=10 \mathrm{~dB}$. Furthermore, the transmission distances of the network are selected as $\left(d_{s r}, d_{r d}, d_{s d}, d_{s p}, d_{r p}\right)=$ $(0.5,0.5,0.7,0.8,0.8)$. It can be observed from Fig. 3 that an increase of $P E R_{T G}$ leads to an increase of the transmission efficiency. This is because, given the other parameters, when transmission rate increases, the transmission efficiency will increase at the cost of also increasing the average PER.

Thirdly, in Fig. 4 and Fig. 5, we make comparisons of outage probability and transmission efficiency for our system utilizing $\mathrm{AMC}$ and relay queue awareness with that of a system without AMC, i.e., operating with Mode 3 of Table I and that of the direct transmission utilizing AMC. Here, we select Mode 3 of Table I, to illustrate performance of a system without AMC as an example. The value of $P E R_{T G}$ and $P_{\max } / N_{0}$ are fixed at $10^{-3}$ and $10 \mathrm{~dB}$, respectively. Furthermore, the transmission distances of the network are selected as $\left(d_{s r}, d_{r d}, d_{s d}, d_{s p}, d_{r p}\right)=(0.5,0.5,0.8,0.8,0.8)$. As expected, in the whole investigated range of interference power-to-noise ratio regime, the system with $\mathrm{AMC}$ together with relay queue awareness policy obtains the best performance.

As a final point, for all the examined scenarios, we can observe that both the outage and transmission efficiency converge to a constant value when $Q / N_{0}$ goes beyond a certain value, for instance, $15 \mathrm{~dB}$. This is because the transmit power of the secondary network not only is constrained by $Q$, but also is constrained by its transmit power limit $P_{\max }$, $P_{s}=\min \left(P_{\max }, Q / X_{s p}\right)$, and $P_{r}=\min \left(P_{\max }, Q / X_{r p}\right)$. When $Q$ is large enough, the transmit powers of S and R only depend on $P_{\max }$. In this case, the transmit powers of $\mathrm{S}$ and $\mathrm{R}$ are fixed at $P_{\max }$. As a result, both the outage probability and transmission efficiency will no longer increase when $Q / N_{0}$ increases.

\section{CONCLUSION}

In this paper, we have analyzed the performance of AMC with queue awareness in cognitive incremental DF relaying systems. In order to maximize the overall spectrum efficiency, a suitable operation mode, including direct and relaying transmission with MCSs, is selected. Furthermore, to avoid buffer overflow at R, the MCS in the relaying transmission is chosen subject to both the SNR of the fading channels and the current state of the buffer. A finite-state Markov chain model is applied to analyze the distribution of the number of packets in the buffer of $\mathrm{R}$ which enables us to derive expressions for key performance indicators such as outage probability and average transmission efficiency of the network. Based on the obtained analysis, numerical results are provided to reveal performance advantages of the cognitive incremental DF relay network with $\mathrm{AMC}$ and queue awareness compared to cognitive relaying without $\mathrm{AMC}$, and conventional direct transmission without AMC.

\section{REFERENCES}

[1] J. Mitola, "Cognitive radio for flexible mobile multimedia communications," in Proc. IEEE International Workshop on Mobile Multimedia Communications, San Diego, US, Nov. 1999, pp. 3-10.

[2] — "Cognitive radio: An integrated agent architecture for software defined radio," Ph.D. dissertation, Royal Institute of Technology, Stockholm, Sweden, 2000.

[3] FCC, "Spectrum policy task force report (et docket no. 02-135)," Nov. 2002. [Online]. Available: hraunfoss.fcc.gov/edocs_public/attachmatch/DOC-228542A1.pdf

[4] Q. Zhang, J. Jia, and J. Zhang, "Cooperative relay to improve diversity in cognitive radio networks," IEEE Commun. Mag., vol. 47, no. 2, pp. 111-117, Feb. 2009.

[5] J. N. Laneman, D. N. C. Tse, and G. W. Wornell, "Cooperative diversity in wireless networks: Efficient protocols and outage behavior," IEEE Trans. Inf. Theory, vol. 50, no. 12, pp. 3062-3080, Dec. 2004.

[6] S. Falahati, A. Svensson, M. Sternad, and T. Ekman, "Adaptive modulation systems for predicted wireless channels," in Proc. IEEE Global Communications Conference, San Francisco, USA, Dec. 2003, pp. 357361.

[7] T. M. C. Chu, H. Phan, and H.-J. Zepernick, "MIMO incremental AF relay networks with TAS/MRC and adaptive modulation," in Proc. IEEE Vehicular Technology Conference, Las Vegas, USA, Sep. 2013, pp. 1-5.

[8] I. S. Gradshteyn and I. M. Ryzhik, Table of Integrals, Series, and Products, 7th ed. Academic Press, 2007.

[9] T. M. C. Chu, H. Phan, and H.-J. Zepernick, "On the performance of underlay cognitive radio networks using $\mathrm{M} / \mathrm{G} / 1 / \mathrm{K}$ queueing model," IEEE Commun. Lett., vol. 17, no. 5, pp. 876-879, May 2013.

[10] M. D. Renzo, F. Graziosi, and F. Santucci, "A comprehensive framework for performance analysis of dual-hop cooperative wireless systems with fixed-gain relays over generalized fading channels," IEEE Trans. Wireless Commun., vol. 8, no. 10, pp. 5060-5074, Oct. 2009.

[11] A. Muller and H. C. Yang, "Dual-hop adaptive packet transmission systems with regenerative relaying," IEEE Trans. Wireless Commun., vol. 9, no. 1, pp. 234-244, Jan. 2010.

[12] Q. Liu, S. Zhou, and G. B. Giannakis, "Cross-layer combining of adaptive modulation and coding with truncated ARQ over wireless links," IEEE Trans. Wireless Commun., vol. 3, no. 5, pp. 1746-1755, Sep. 2004.

[13] Y. Zhang, Y. Chang, S. Wang, and D. Yang, "Performance of selection combining amplify-and-forward relaying with adaptive modulation over Nakagami-m fading channels," in Proc. IEEE Vehicular Technology Conference, San Francisco, USA, Sep. 2011, pp. 1-5. 